\title{
Optimal Cross Futures Hedging Under Regime Switching Time-Varying Correlated Jump Dynamic
}

\author{
Hsiang-Tai Lee ${ }^{1}$ \\ ${ }^{1}$ Professor, Department of Banking and Finance, 1, University Rd., Puli, Nantou Hsien, \\ National Chi Nan University, Taiwan 54561
}

\begin{abstract}
A bivariate regime switching time-varying correlated jump model (RSTVCJ) is proposed for optimal hedging. Most of the existing discrete time hedging models does not consider simultaneously the jump risk and regime switching risk. Two recent articles (Lee 2009a, 2015) suggest jump switching models that possess both of these risks. These models, however, assume a common jump for spot and futures returns. This might be too restrictive because spot and futures returns are normally not perfectly correlated especially for the cases of cross hedging where the corresponding futures contract is not available for the underlying spot. In this paper, I release this common jump assumption and envision a regime switching time-varying correlated jump model for optimal hedging. In the proposed RSTVCJ, the spot and futures returns possess both self-owned and correlated jumps and the correlated jump is captured via a regime switching spillover factor. We illustrate the usefulness of RSTVCJ by performing the exercise of hedging jet fuel spot holding with crude oil futures. We compare the performance of RSTVCJ with its nested models including common jump (CJ), state-independent correlated jump (SICJ), regime switching correlated jump (RSCJ). Empirical results show that RSCJ has the best outof-sample hedging performance. Regime switching correlated jump is superior to its state-independent or common jump counterparts. Adding time-varying dynamic to the jump process, however, does not further improve the hedging effectiveness.
\end{abstract}

Keywords: Regime switching; correlated time-varying jump; cross hedging

\section{Introduction}

The benefits of fitting the spot and futures returns with more flexible distribution or specifications in futures hedging are widely investigated in the past two decades. Apart from the conventional static hedging with ordinary least square (OLS) method, the most popular futures hedging strategies are implemented with a wide array of multivariate GARCH models to estimate the time-varying minimum variance hedge ratio (MVHR) (Baillie and Myers, 1991; Kroner and Sultan, 1993; Park and Switzer, 1995; Gagnon and Lypny, 1998; Brooks et al., 2002; Alexander and Barbosa, 2008; Ederington and Salas, 2008). These models, however, capture mainly the continuous variations in the covariance structures of the spot and futures returns.

To capture the sudden jump risk in the covariance structure of spot and futures returns, a number of studies apply jump models for futures hedging. Chan and Young (2006) proposed a bivariate GARCH-jump model with autoregressive jump intensity (Chan and Maheu, 2002) to capture the features of the joint distribution of copper cash and futures returns. Based on the results of within-sample and out-of-sample hedging exercises, they report significant gains of applying a time-varying optimal hedging strategy that incorporates the information from the common jump dynamics. Chan (2008) apply a dynamic hedging strategy based on a bivariate GARCH-jump model augmented with autoregressive jump intensity for hedging foreign 
currency jump risk. It finds significant common jump components in the currency spot rate and futures basis with jump sizes response asymmetrical to futures basis changes. Chan (2010) applies a bivariate GARCH model augmented with a common jump component to manage currency risk. It finds significant common jump components in the British pound spot and futures rates and shows that incorporating common jump dynamics substantially reduce daily and weekly portfolio risk. Generally speaking, incorporating common jump risks for spot and futures returns improves futures hedging effectiveness. Although these common jump models are more flexible than jump-free models, it ignores the property of regime switching (Alizadeh and Nomikos, 2004; Lee and Yoder, 2007a) and it assumes a common jump dynamic for both spot and futures returns which might be too restrictive because spot and futures returns are normally not perfectly correlated. ${ }^{1}$

Another strand in the line of optimal futures hedging literature focuses on the regimeshifting risk in the joint distribution of spot and futures returns. The existence of regime-shifting relationship between spot and futures data series is demonstrated in a series papers of Sarno and Valente (2000, 2005a, 2005b). Based on these findings, many studies start to estimate the so-called state-dependent time-varying minimum variance hedge ratio by proposing a wide array of multivariate Markov regime switching models (Alizadeh and Nomikos, 2004; Lee and Yoder, 2007a, 2007b; Alizadeh et al., 2008; Lee, 2010; Sheu and Lee, 2014). Lee and Yoder (2007a) apply a regime switching BEKK GARCH for estimating the state-dependent timevarying MVHR. Alizadeh et al. (2008) further suggest a regime switching BEKK GARCH with cointegration relationship between spot and futures returns for implementing energy futures hedging strategy. For explicitly modeling the correlation dynamic of spot and futures returns, Lee and Yoder (2007b) suggest a regime switching varying correlation GARCH, and Lee adopts a copula-based regime-switching GARCH model (2009b) and a regime switching dynamic conditional correlation GARCH (2010) for futures hedging. To release the assumption of common switching dynamic for spot and futures returns, Sheu and Lee (2014) suggest a multi-chain Markov regime switching GARCH model for futures hedging. A general finding is that incorporating regime switching effect improves futures hedging effectiveness.

Most of the existing hedging models to date consider the jump risk and regime-shifting risk separately. Two recent articles (Lee 2009a, 2015) suggest jump switching models that possess both of these risks. ${ }^{2}$ (Lee 2009a) develops a Markov regime switching Generalized Orthogonal GARCH model with conditional jump dynamics (JSGO) for hedging FTSE 100 spot returns and finds significant hedging gains of JSGO compared to its jump-free and stateindependent counterparts. (Lee 2015) suggests a multi-chain regime switching jump spillover GARCH $(M C R S J)$ model to investigate the spillover behavior from stock index futures to stock sectors. Cross hedging exercises show that taking account of volatility spillover and statedependent jump dynamic improves futures hedging performance. These models, however, assume a common jump for spot and futures returns which might be too restrictive. For cross

1 Chan (2003) suggests a correlated bivariate poisson jump model for foreign exchange. The model, however, is state-independent and is not applied to futures hedging.

2 Although Chan and Young (2009) and Sheu and Lee (2012) also suggest jump switching models, these models are univariate and do not consider the correlated jump dynamic across asset returns. 
hedging applications, such as cross hedging the crude oil risk of non-energy commodities (Sheu et al., 2015), hedging the single stock with ADR (Lee and Tsang, 2011), hedging the stock sectors price risk with stock index futures (Lee and Ko, 2010), hedging stock sector risk with credit default swap (Ratner and Chiu, 2013) and so on, the corresponding hedging asset has quite different price dynamic from the underlying spot and the assumption of common jump for both assets will be far away from realistic. In this paper, I attempt to release this common jump assumption and envision a regime switching time-varying correlated jump model for optimal hedging. The proposed RSTVCJ model allows the spot and futures returns to possess both self-owned and correlated jumps and the correlated jump is captured via a regime switching spillover factor. In this paper, cross hedging exercises on jet fuel spot holding with crude oil futures will be performed to justify the usefulness of RSTVCJ.

\section{Regime switching time-varying correlated jump (RSTVCJ) model}

The main feature of the proposed regime switching time-varying correlated jump model (RSTVCJ) that distinguishes itself from previous regime switching jump model is that different assets could possess simultaneously the self-owned jumps and correlated jumps and the correlated jump is captured via a regime switching spillover factor. The specification of RSTVCJ is given below:

Let $\mathbf{R}_{t}$ be a $2 \times 1$ vector of spot and futures returns with conditional mean equation

$$
\mathbf{R}_{t}=\left[\begin{array}{c}
r_{c, t} \\
r_{f, t}
\end{array}\right]=\boldsymbol{\mu}_{s_{t}}+\mathbf{v}_{t, s_{t}}=\boldsymbol{\mu}_{s_{t}}+\boldsymbol{\varepsilon}_{t, s_{t}}+\mathbf{J}_{t, s_{t}},
$$

where $r_{c, t}$ and $r_{f, t}$ are respectively the spot and futures returns and the subscription $s_{t}=\{1,2\}$ stands for the state variable assumed to follow a first-order, two-state Markov process with a logistic state transition probabilities given by

$$
\begin{aligned}
& \operatorname{Pr}\left(s_{t}=1 \mid s_{t-1}=1\right)=\frac{\exp \left(p_{0}\right)}{1+\exp \left(p_{0}\right)}, \\
& \operatorname{Pr}\left(s_{t}=2 \mid s_{t-1}=2\right)=\frac{\exp \left(q_{0}\right)}{1+\exp \left(q_{0}\right)},
\end{aligned}
$$

where $p_{0}$ and $q_{0}$ are unconstrained parameters to be estimated along with system parameters.

$$
\boldsymbol{\mu}_{s_{t}}=\left[\begin{array}{c}
\mu_{c, s_{t}} \\
\mu_{f, s_{t}}
\end{array}\right] \text { is a vector of state-dependent unconditional mean returns, } \boldsymbol{\varepsilon}_{t, s_{t}}=\left[\begin{array}{c}
\varepsilon_{c, t, s_{t}} \\
\varepsilon_{f, t, s_{t}}
\end{array}\right] \text { is }
$$

a bivariate state-dependent residual vector and $\mathbf{J}_{t, s_{t}}=\left[\begin{array}{l}J_{c, t, s_{t}} \\ J_{f, t, s_{t}}\end{array}\right]$ is a bivariate state-dependent jump component vector. The state-dependent residual vector $\boldsymbol{\varepsilon}_{t, s_{t}}$ is assumed to be normally distributed given by

$$
\boldsymbol{\varepsilon}_{t, s_{t}} \mid \psi_{t-1} \sim N\left(\mathbf{0}, \widetilde{\mathbf{H}}_{s_{t}}\right) .
$$




\section{International Conference on \\ MANAGEMENT, ECONOMICS \& FINANCE}

where $\psi_{t-1}$ is the information set available at time $t-1$ and the state-dependent covariance matrix $\tilde{\mathbf{H}}_{s_{t}}$ is given by

$$
\begin{aligned}
\widetilde{\mathbf{H}}_{s_{t}} & =\left[\begin{array}{ll}
h_{c, t, s_{t}} & h_{c f, t, s_{t}} \\
h_{c f, t, s_{t}} & h_{f, t, s_{t}}
\end{array}\right]=\left[\begin{array}{cc}
\sqrt{h_{c, t, s_{t}}} & 0 \\
0 & \sqrt{h_{f, t, s_{t}}}
\end{array}\right]\left[\begin{array}{cc}
1 & \rho_{c f, s_{t}} \\
\rho_{c f, s_{t}} & 1
\end{array}\right]\left[\begin{array}{cc}
\sqrt{h_{c, t, s_{t}}} & 0 \\
0 & \sqrt{h_{f, t, s_{t}}}
\end{array}\right] \\
& =\mathbf{D}_{t, s_{t}} \boldsymbol{\Gamma}_{t, s_{t}} \mathbf{D}_{t, s_{t}} .
\end{aligned}
$$

In equation (5), $h_{c, t, s_{t}}$ and $h_{f, t, s_{t}}$ are respectively the state-dependent volatilities of spot and futures returns assumed to follow regime switching $\operatorname{GARCH}(1,1)$ processes defined as

$$
\begin{aligned}
& h_{c, t, s_{t}}=\gamma_{c, s_{t}}+\alpha_{c, s_{t}} v_{c, t-1, s_{t}}^{2}+\beta_{c, s} h_{c, t-1, s_{t}}, \\
& h_{f, t, s_{t}}=\gamma_{f, s_{t}}+\alpha_{f, s_{t}} v_{f, t-1, s_{t}}^{2}+\beta_{f, s} h_{f, t-1, s_{t}} .
\end{aligned}
$$

As for the jump component, Lee (2009a) assumes a state-independent common jump for both spot and futures returns. Although Lee (2015) suggests a model with state-dependent jump, it still assumes a common jump for both spot and futures returns. The proposed RSTVCJ possesses both self-owned and correlated jumps with specification depicted below:

The jump component $\mathbf{J}_{t, s_{t}}$ is a 2-dimensioned state-dependent vector defined as

$$
\mathbf{J}_{t, s_{t}}\left|\psi_{t-1}=\left[\begin{array}{c}
J_{c, t, s_{t}} \\
J_{f, t, s_{t}}
\end{array}\right]\right| \psi_{t-1} \sim N\left(\mathbf{0}, \boldsymbol{\Sigma}_{J, s_{t}}\right),
$$

where $\Sigma_{J, s_{t}}$ is the state-dependent jump covariance matrix and the jump components are defined as $J_{c, t, s_{t}}=J_{c, t, s_{t}}^{*}+\eta_{s_{t}} J_{f, t, s_{t}}^{*}$ and $J_{f, t, s_{t}}=J_{f, t, s_{t}}^{*}$. The jump components $J_{c, t, s_{t}}^{*}$ and $J_{f, t, s_{t}}^{*}$ capture respectively the spot and futures self-owned jump dynamics and the term $\eta_{s_{t}} \boldsymbol{J}_{f, t, s_{t}}^{*}$ captures the correlated jump via a state-dependent spillover factor $\eta_{s_{t}}$. These jump components enters the mean equation with an expected value of zero achieved by subtracting the expected values from the series of random jumps defined as

$$
\begin{aligned}
& J_{c, t, s_{t}}^{*}=\sum_{k=1}^{n_{c, t, s_{t}}} Y_{k, c, t}-E\left[\sum_{k=1}^{n_{c, t, s_{t}}} Y_{k, c, t}\right], \\
& J_{f, t, s_{t}}^{*}=\sum_{k=1}^{n_{f, t, s_{t}}} Y_{k, f, t}-E\left[\sum_{k=1}^{n_{f, t, s_{t}}} Y_{k, f, t}\right] .
\end{aligned}
$$

The state-dependent discrete counting processes governing the number of jumps that arrives between time $t-1$ and $t$ for the spot and futures returns are denoted respectively as $n_{c, t, s_{t}}$ and $n_{f, t, s_{t}}$ and are constructed as a sum of a series of random variables $Y^{\prime} s$ given by

$$
\sum_{k=1}^{n_{c, t, s_{t}}} Y_{k, c, t}=Y_{1, c, t}+Y_{2, c, t}+\cdots+Y_{n_{c, t, s,}, c, t}
$$




\section{International Conference on \\ MANAGEMENT, ECONOMICS \& FINANCE}

$$
\sum_{k=1}^{n_{f, t, s_{t}}} Y_{k, f, t}=Y_{1, f, t}+Y_{2, f, t}+\cdots+Y_{n_{f, t, s,}, f, t},
$$

where the random variable $Y^{\prime} s$ stand for jump size governed by a normal distribution with constant mean and constant volatility given by

$$
\begin{aligned}
Y_{c, t} & \sim N\left(\tau_{c}, \delta_{c}^{2}\right), \\
Y_{f, t} & \sim N\left(\tau_{f}, \delta_{f}^{2}\right) .
\end{aligned}
$$

The means and volatilities are assumed to remain the same across time but different across assets. The discrete counting processes $n_{c, t, s_{t}}$ and $n_{f, t, s_{t}}$ are distributed as a Poisson random variable with the jump intensity parameter $\lambda_{t}>0$ and density

$$
\begin{aligned}
& P\left(n_{c, t, s_{t}}=i \mid s_{t}=k, \psi_{t-1}\right)=\frac{\exp \left(-\lambda_{c, s_{t}}\right) \lambda_{c, s t}^{i}}{i !}, i=0,1, \cdots . \\
& P\left(n_{f, t, s_{t}}=j \mid s_{t}=k, \psi_{t-1}\right)=\frac{\exp \left(-\lambda_{f, s_{t}}\right) \lambda_{f, s_{t}}^{j}}{j !}, i=0,1, \cdots .
\end{aligned}
$$

The jump intensities $\lambda_{i, t, s_{t}}=E\left[n_{i, t, s_{t}} \mid \psi_{t-1}\right], i \in\{c, f\}$ are assumes to be time-varying given by

$$
\lambda_{i, t, s_{t}}=\lambda_{i, 0, s_{t}}+a_{i, s_{t}} \lambda_{t-1, s_{t}}+b_{i, s_{t}} \xi_{t-1, s_{t}}
$$

where $\xi_{t-1, s_{t}}$ is the jump intensity residual defined as

$$
\begin{aligned}
& \xi_{i, t-1, s_{t}}=E\left[n_{i, t-1, s_{t}} \mid \psi_{t-1}\right]-\lambda_{i, t-1, s_{t}} \\
& =\sum_{i=0}^{\infty} i P\left(n_{i, t, s_{f, t-1}}=i \mid s_{t-1}=k, \psi_{t-1}\right)-\lambda_{i, t-1, s_{t}} .
\end{aligned}
$$

With these definitions, the return vector $\mathbf{R}_{t}$ is normally distributed with covariance matrix $\mathbf{H}_{t, s_{t}}$ given by

$$
\mathbf{H}_{t, s_{t}}=\widetilde{\mathbf{H}}_{s_{t}}+\boldsymbol{\Sigma}_{J, s_{t}},
$$

where the regular covariance matrix $\tilde{\mathbf{H}}_{s_{t}}$ is defined in equation (5), the jump covariance matrix $\Sigma_{J, s_{t}}$ can be shown as

$$
\boldsymbol{\Sigma}_{J_{t, s i}}=\left[\begin{array}{cc}
\lambda_{c, s_{t}}\left(\delta_{c}^{2}+\tau_{c}^{2}\right)+\eta_{s_{t}}^{2} \lambda_{f, s_{s}}\left(\delta_{f}^{2}+\tau_{f}^{2}\right) & \eta_{s_{t}} \lambda_{f, s_{t}}\left(\delta_{f}^{2}+\tau_{f}^{2}\right) \\
\eta_{s_{t}} \lambda_{f, s_{t}}\left(\delta_{f}^{2}+\tau_{f}^{2}\right) & \lambda_{f, s_{t}}\left(\delta_{f}^{2}+\tau_{f}^{2}\right)
\end{array}\right],
$$




\section{International Conference on \\ MANAGEMENT, ECONOMICS \& FINANCE}

and the state-dependent jump correlation coefficient of spot and futures returns $\rho_{t, s_{t}}$ can be calculated as

$$
\rho_{J, t, s_{t}}=\frac{\eta_{s_{t}} \lambda_{f, s_{t}}\left(\delta_{f}^{2}+\tau_{f}^{2}\right)}{\sqrt{\left[\lambda_{c, s_{t}}\left(\delta_{c}^{2}+\tau_{c}^{2}\right)+\eta_{s_{t}}^{2} \lambda_{f, s_{t}}\left(\delta_{f}^{2}+\tau_{f}^{2}\right)\right]\left[\lambda_{f, s_{t}}\left(\delta_{f}^{2}+\tau_{f}^{2}\right)\right]}} .
$$

Accordingly, the state-dependent total correlation is given by

$$
\rho_{t, s_{t}}=\frac{h_{c f, t, s_{t}}+\eta_{s_{t}} \lambda_{f, s_{t}}\left(\delta_{f}^{2}+\tau_{f}^{2}\right)}{\sqrt{\left[h_{c, t, s_{t}}+\lambda_{c, s_{t}}\left(\delta_{c}^{2}+\tau_{c}^{2}\right)+\eta_{s_{t}}^{2} \lambda_{f, s_{t}}\left(\delta_{f}^{2}+\tau_{f}^{2}\right)\right]\left[h_{f, t, s_{t}}+\lambda_{f, s_{t}}\left(\delta_{f}^{2}+\tau_{f}^{2}\right)\right]}} .
$$

Equations (1)-(18) constitute the specification of RSTVCJ. The detail estimation procedure of RSTVCJ is depicted in the next section.

\section{The estimation procedure of the regime switching time-varying correlated jump (RSTVCJ) model}

The regime switching time-varying correlated jump (RSTVCJ) model is estimated by maximizing the following log-likelihood function

$$
L(\Theta)=\sum_{t=1}^{T} \log \left[f\left(\mathbf{R}_{t} \mid \psi_{t-1}\right)\right],
$$

where $T$ is the total number of observations, $\Theta$ is a set of unknown parameters, $f\left(\mathbf{R}_{t} \mid \psi_{t-1}\right)$ is the mixture distribution weighted by the conditional joint probabilities of state variables. The conditional likelihood of each observation at time $t$ is given by

$$
\begin{gathered}
f\left(\mathbf{R}_{t} \mid \psi_{t-1}\right)=\sum_{k=0}^{1} \sum_{j=0}^{\infty} \sum_{i=0}^{\infty} f\left(\mathbf{R}_{t}, n_{c, t, s_{t}}=i, n_{f, t, s_{t}}=j, s_{t}=k \mid \psi_{t-1}\right) \\
=\sum_{k=0}^{1} \sum_{j=0}^{\infty} \sum_{i=0}^{\infty} f\left(\mathbf{R}_{t} \mid n_{c, t, s_{t}}=i, n_{f, t, s_{t}}=j, s_{t}=k, \psi_{t-1}\right) \times P\left(n_{c, t, s_{t}}=i, n_{f, t, s_{t}}=j \mid s_{t}=k, \psi_{t-1}\right) \times P\left(s_{t}=k \mid \psi_{t-1}\right)
\end{gathered}
$$

where $f\left(\mathbf{R}_{t} \mid n_{c, t, s_{t}}=i, n_{f, t, s_{t}}=j, s_{t}=k, \psi_{t-1}\right)$ denotes the conditional density of return vector given that $i$ jumps occur in the spot return, $j$ jumps occur in the futuers return and state variable is in the $k$ state, $P\left(n_{c, t, s_{t}}=i, n_{f, t, s_{t}}=j \mid s_{t}=k, \psi_{t-1}\right)$ is the joint distribution of two discrete counting processes $n_{c, t, s_{t}}$ and $n_{f, t, s_{t}}$ and $P\left(s_{t}=k \mid \psi_{t-1}\right)$ is the conditional regime probability of being in state $k$. To complete the likelihood of RSTVCJ, a jump switching algorithm has to be applied. Lee's filter (Lee, 2009a, Sheu and Lee, 2012) is an intervening filter of Hamilton filter (Hamilton, 1989) and Chan and Maheu's filter (Chan and Maheu, 2002). The steps of generalized filtering algorithm for RSTVCJ with correlated jump components are depicted below:

(1) projects both the state and jump probabilities

$$
P\left(s_{t}=i \mid \psi_{t-1}\right)=\sum_{j=0}^{1} P\left(s_{t}=i \mid s_{t-1}=j\right) \times P\left(s_{t-1}=j \mid \psi_{t-1}\right),
$$




$$
P\left(n_{c, t, s_{t}}=i, n_{f, t, s_{t}}=j \mid s_{t}=k, \psi_{t-1}\right)=P\left(n_{c, t, s_{t}}=i \mid s_{t}=k, \psi_{t-1}\right) \times P\left(n_{f, t, s_{t}}=j \mid s_{t}=k, \psi_{t-1}\right),
$$

where $P\left(s_{t}=i \mid s_{t-1}=j\right)$ is the regime transition probability defined in equations (2) and (3) and $P\left(n_{c, t, s_{t}}=i \mid s_{t}=k, \psi_{t-1}\right)$ and $P\left(n_{f, t, s_{t}}=j \mid s_{t}=k, \psi_{t-1}\right)$ are Poisson distributed statedependent probabilities defined in equations (15) and (16).

(2) Evaluate the jump and regime dependent likelihood

The assumptions in (4) and (8) imply that the joint distribution of spot and futures returns conditional on the most recent information set, state $k, i$ spot jumps and $j$ futures jumps is normally distributed given by

$$
f\left(\mathbf{R}_{t} \mid n_{c, t, s_{t}}=i, n_{f, t, s_{t}}=j, s_{t}=k, \psi_{t-1}\right)=\frac{1}{2 \pi}\left|\mathbf{H}_{t, i, j, k}\right|^{-\frac{1}{2}} \exp \left\{-\frac{1}{2} \mathbf{v}_{t, i, j, k}^{T} \mathbf{H}_{t, i, j, k}^{-1} \mathbf{v}_{t, i, j, k}\right\}
$$

$$
=\frac{1}{2 \pi}\left|\tilde{\mathbf{H}}_{t, k}+\boldsymbol{\Sigma}_{J_{t, i, j, k}}\right|-\frac{1}{2} \times \exp \left\{-\frac{1}{2} \mathbf{v}_{t, i, j, k}^{T}\left[\tilde{\mathbf{H}}_{t, k}+\boldsymbol{\Sigma}_{J_{t, i, j, k}}\right]^{-1} \mathbf{v}_{t, i, j, j, k}\right\},
$$

where the jump and regime dependent residual vector can be shown as

$$
\mathbf{v}_{t, i, j, k}=\left[\begin{array}{c}
r_{c, t}-\mu_{c, k}-i \tau_{c}+\lambda_{c, k} \tau_{c}-\eta_{k}\left(j \tau_{f}-\lambda_{f, k} \tau_{f}\right) \\
r_{f, t}-\mu_{f, k}-j \tau_{f}+\lambda_{f, k} \tau_{f}
\end{array}\right],
$$

and the jump and regime dependent covariance matrix is given by

$$
\boldsymbol{\Sigma}_{J_{t, i, j, k}}=\left[\begin{array}{cc}
i \delta_{c}^{2}+\eta_{k}^{2} j \delta_{f}^{2} & \eta_{k} j \delta_{f}^{2} \\
\eta_{k} j \delta_{f}^{2} & j \delta_{f}^{2}
\end{array}\right] .
$$

(3) Evaluate the mixture likelihood

The mixture likelihood is given by

$$
f\left(\mathbf{R}_{t} \mid \psi_{t-1}\right)=\sum_{k=0}^{1} \sum_{j=0}^{\infty} \sum_{i=0}^{\infty} f\left(\mathbf{R}_{t}, n_{c, t, s_{t}}=i, n_{f, t, s_{t}}=j, s_{t}=k \mid \psi_{t-1}\right),
$$

where $f\left(\mathbf{R}_{t}, n_{c, t, s_{t}}=i, n_{f, t, s_{t}}=j, s_{t}=k \mid \psi_{t-1}\right)$ is the joint distribution of return vector, spot jump, futures jump and state variable. ${ }^{3}$

(4) Update both state and jump probabilities

$$
\begin{aligned}
& P\left(s_{t}=k \mid \psi_{t}\right)=\frac{f\left(\mathbf{R}_{t} \mid s_{t}=k, \psi_{t-1}\right) \times P\left(s_{t}=k \mid \psi_{t-1}\right)}{f\left(\mathbf{R}_{t} \mid \psi_{t-1}\right)}, \\
& P\left(n_{c, t, s_{t}}=i, n_{f, t, s_{t}}=j \mid s_{t}=k, \psi_{t}\right)
\end{aligned}
$$

3 Equation (32) involves an infinite sum over the possible number of jumps $n_{t}$ which is not feasible in estimation. According to Lee (2009a), in practice, the maximum number of jumps $\eta$ is truncated at a large enough value such that the probability of $\eta$ or more jumps to the machine precision is 0 and the likelihood and the parameter estimate do not change. 


$$
=\frac{f\left(R_{t} \mid n_{c, t, s_{t}}=i, n_{f, t, s_{t}}=j, s_{t}=i, \psi_{t-1}\right) \times P\left(n_{c, t, s_{t}}=i \mid s_{t}=k, \psi_{t-1}\right) \times P\left(n_{f, t, s_{t}}=j \mid s_{t}=k, \psi_{t-1}\right)}{f\left(R_{t} \mid s_{t}=i, \psi_{t-1}\right)}
$$

where $f\left(R_{t} \mid s_{t}=i, \psi_{t-1}\right)$ can be shown as

$f\left(R_{t} \mid s_{t}=i, \psi_{t-1}\right)$

$=\sum_{i=0}^{\infty} \sum_{j=0}^{\infty} f\left(R_{t} \mid s_{t}=i, n_{c, t, s_{t}}=i, n_{f, t, s_{t}}=j, \psi_{t-1}\right) \times P\left(n_{c, t, s_{t}}=i \mid s_{t}=k, \psi_{t-1}\right) \times P\left(n_{f, t, s_{t}}=j \mid s_{t}=k, \psi_{t-1}\right)$

(5) Calculate the weighted average residuals, volatilities, covariance, jump intensity and spillover factor for feasible estimation. The procedure of recombining is depicted below.

(6) Iterate (1) to (5) until the end of the sample and the likelihood is obtained as given in equation (23).

When a recursive process is subject to regime switching, the recursive nature of the process makes the model intractable (Cai, 1994; Hamilton and Susmel, 1994; Gray, 1996, Lee et al., 2006, Lee and Yoder, 2007a). To complete the likelihood in (23), step (5) requires a recombining procedure to solve this path-dependency problem to make the RSTVCJ tractable. The residuals of the spot return $v_{c, t}$ and futures return $v_{f, t}$ can be recombined using the following equations:

$$
\begin{aligned}
& v_{c, t}=r_{c, t}-E\left[r_{c, t} \mid \psi_{t-1}\right]=r_{c, t}-\left[p_{1 t}\left(\mu_{c, 1}\right)+\left(1-p_{1 t}\right)\left(\mu_{c, 2}\right)\right] \\
& v_{f, t}=r_{f, t}-E\left[r_{f, t} \mid \psi_{t-1}\right]=r_{f, t}-\left[p_{1 t}\left(\mu_{f, 1}\right)+\left(1-p_{1 t}\right)\left(\mu_{f, 2}\right)\right]
\end{aligned}
$$

where $p_{1 t}$ is the conditional probability of being in regime 1 at time $t$. The conditional variance of futures return can be recombined using the following equation:

$$
\begin{aligned}
h_{f, t \mid t-1}= & \operatorname{Var}\left(\varepsilon_{f, t} \mid \psi_{t-1}\right)=\operatorname{Var}\left(r_{f, t} \mid \psi_{t-1}\right)-\operatorname{Var}\left(J_{f, t} \mid \psi_{t-1}\right), \\
& =p_{1 t}\left(\mu_{c, 1}^{2}+h_{c, t, 1}\right)+\left(1-p_{1 t}\right)\left(\mu_{c, 2}^{2}+h_{c, t, 2}\right)-\left[p_{1 t}\left(\mu_{f, 1}\right)+\left(1-p_{1 t}\right)\left(\mu_{f, 2}\right)\right]^{2}
\end{aligned}
$$

The conditional variance of spot return can be recombined using the following equation:

$$
\begin{aligned}
h_{c, t \mid t-1} & =\operatorname{Var}\left(\varepsilon_{c, t} \mid \psi_{t-1}\right)=\operatorname{Var}\left(r_{c, t} \mid \psi_{t-1}\right)-\operatorname{Var}\left(J_{c, t, s_{t}}^{*}+\eta_{s_{t}} J_{f, t, s_{t}}^{*} \mid \psi_{t-1}\right), \\
& =p_{1 t}\left(\mu_{c, 1}^{2}+h_{c, t, 1}\right)+\left(1-p_{1 t}\right)\left(\mu_{c, 2}^{2}+h_{c, t, 2}\right) .
\end{aligned}
$$

As for the recombination of the covariance of spot and futures returns, the following weighted average is applied:

$$
h_{c f, t}=\operatorname{Cov}\left(r_{c, t}, r_{f, t} \mid \psi_{t-1}\right)=E\left[r_{c, t} r_{f, t} \mid \psi_{t-1}\right]-E\left[r_{c, t} \mid \psi_{t-1}\right] E\left[r_{f, t} \mid \psi_{t-1}\right],
$$

where 


$$
E\left[r_{c, t} \mid \psi_{t-1}\right]=\left[p_{1 t}\left(\mu_{c, 1}\right)+\left(1-p_{1 t}\right)\left(\mu_{c, 2}\right)\right]
$$

$$
E\left[r_{f, t} \mid \psi_{t-1}\right]=\left[p_{1 t}\left(\mu_{f, 1}\right)+\left(1-p_{1 t}\right)\left(\mu_{f, 2}\right)\right]
$$

and

$$
\begin{aligned}
E\left[r_{c, t} r_{f, t} \mid \psi_{t-1}\right] & =p_{1 t}\left[\mu_{c, 1} \mu_{f, 1}+h_{c f, t, 1}+\eta_{c, 1} \lambda_{f, 1}\left(\delta_{f}^{2}+\tau_{f}^{2}\right)\right] \\
& +\left(1-p_{1 t}\right)\left[\mu_{c, 2} \mu_{f, 2}+h_{c f, t, 2}+\eta_{c, 2} \lambda_{f, 2}\left(\delta_{f}^{2}+\tau_{f}^{2}\right)\right] .
\end{aligned}
$$

The time-varying jump intensity $\lambda_{i, t}$ and $\eta_{i, t}$ can be recombined as

$$
\lambda_{i, t}=p_{1 t} \lambda_{i, t, 1}+\left(1-p_{1 t}\right) \lambda_{i, t, 2}, i \in\{c, f\},
$$

$$
\eta_{t}=p_{1 t} \eta_{t, 1}+\left(1-p_{1 t}\right) \eta_{t, 2}
$$

The unknown parameters in RSTVCJ are $\Theta=\left\{p_{0}, q_{0}, \mu_{c, s_{c, t}}, \mu_{f, s_{f, t}}, \gamma_{c, s_{c, t}}, \gamma_{f, s_{f, t}}, \alpha_{c, s_{c, t}}\right.$, $\left.\alpha_{f, s_{f, t}}, \beta_{c, s_{c, t}}, \beta_{f, s_{f, t}}, \tau_{c}, \delta_{c}, \tau_{f}, \delta_{f}, \lambda_{c, 0, s_{t}}, a_{c, s_{t}}, b_{c, s_{t}}, \lambda_{f, 0, s_{t}}, a_{f, s_{t}}, b_{f, s_{t}}, \rho_{s_{t}}\right\}$ for $s_{t} \in\{1,2\}$, which is estimated via maximum likelihood estimation.

\section{Measuring hedging performance, data description and empirical results}

The proposed bivariate regime switching time-varying correlated jump model (RSTVCJ) is applied for optimal futures hedging. The focus of this paper will be applications of cross hedging, where common jump is unlikely to be realistic and modeling simultaneously the selfowned and correlated jump might be beneficial. To implement futures hedging strategy, the $R S T V C J$ estimates of time-varying minimum variance hedge ratio denoted as $\chi_{t \mid t-1}$ is given by

$$
\chi_{t} \mid \psi_{t-1}=\frac{\operatorname{Cov}\left(r_{c, t}, r_{f, t} \mid \psi_{t-1}\right)}{\operatorname{Var}\left(r_{f, t} \mid \psi_{t-1}\right)}=\frac{h_{c f, t}+\eta \lambda_{f}\left(\delta_{f}^{2}+\tau_{f}^{2}\right)}{h_{f, t, s_{t}}+\lambda_{f}\left(\delta_{f}^{2}+\tau_{f}^{2}\right)}
$$

where $h_{f, t}$ and $h_{c f, t}$ are the conditional variance of futures returns and conditional covariance of spot and futures returns calculated respectively with equations (38) and (40), and the spillover factor and time-varying jump intensity are calculated respectively with equations (44) and (45). The hedger chooses a hedging strategy that minimizes the variance of hedged portfolio return $r_{p, t}$ measured with the hedging effectiveness $(H E)$ given by

$$
H E=\frac{\operatorname{Var}\left(r_{c, t}\right)-\operatorname{Var}\left(r_{p, t}\right)}{\operatorname{Var}\left(r_{c, t}\right)} \times 100
$$


where $r_{p, t}=r_{c, t}-\hat{\chi}_{t \mid t-1} r_{f, t}$ is the return on hedged portfolio, $\hat{\chi}_{t \mid t-1}$ is the estimated hedge ratio estimated at time $t-1$ and to be held over the time period $[t-1, t], \operatorname{Var}\left(r_{p, t}\right)$ and $\operatorname{Var}\left(r_{c, t}\right)$ are respectively the variances of hedged portfolio and unhedged spot position. Further taking account of the hedged portfolio return, hedger will choose a hedging strategy that maximizes the expected utility. The mean-variance expected utility function (Kroner and Sultan, 1993, Alizadeh and Nomikos, 2004, Lee, 2010, Sheu and Lee, 2014) is applied in this paper to compare the hedging performance of alternatively models:

$$
E\left[U\left(r_{p, t}\right) \mid \psi_{t-1}\right]=E\left[r_{p, t} \mid \psi_{t-1}\right]-\kappa \operatorname{Var}\left(r_{p, t} \mid \psi_{t-1}\right)
$$

where $\kappa$ stands for the coefficient of absolute risk aversion.

The usefulness of the proposed RSTVCJ is illustrated with the exercise of cross hedging jet fuel spot holding with crude oil futures. Weekly jet fuel prices and nearby futures prices of crude oil for the period of January 1991 to December 2016 obtained from Datastream is applied. The returns are computed as the changes in the natural logarithms of prices multiplied by 100 . We use the data from January 1991 to December 2015 for in-sample parameters estimation and the remaining data are used for out-of-sample analysis. Table I shows the summary statistics of the level and returns series of jet fuel spot and crude oil futures. Both jet fuel spot and crude oil futures have the same return of $0.02 \%$ which is positive but quite small. The jet fuel spot return has a volatility of $5.02 \%$ and crude oil futures return has a volatility of $4.47 \%$. According to the Skewness, leptokurtosis, and significant Jarque-Bera statistics, unconditional distribution of jet fuel spot and crude oil futures returns is asymmetric, fat-tailed, and non-Gaussian. This shows the importance of applying a more flexible regime switching correlated jump model for capturing the dynamic of jet fuel spot and crude oil futures returns.

Table II shows the estimates of unknown parameters of regime switching time-varying correlated jump model (RSTVCJ) and two its nested models, regime switching correlated jump model (RSCJ) without autoregressive jump intensity dynamic and state-independent correlated jump model $(S I C J)$ with no regime switching effect in the correlated jump dynamic. To estimate the jump dynamic, the maximum number of jumps $\eta=10$ was selected as the truncation points. In the volatility equation, $\alpha^{\prime} s, \beta^{\prime} s$ and $\gamma^{\prime} s$ are the $\operatorname{GARCH}(1,1)$ parameters of regular volatility dynamic. For a given $\alpha$ and $\beta$, higher $\gamma$ indicates a higher steady state volatility. Based on the estimates of RSTVCJ, $\gamma$ has a higher value in state 2 for both spot and futures returns. $\gamma^{\prime} s$ are respectively equal to 0.212 and 5.022 in state 1 and state 2 for spot return and are respectively equal to 0.925 and 2.697 in state 1 and state 2 for futures return. State 2 is the higher volatility state. The term $\alpha+\beta$ in the volatility equation measures the volatility persistence. The $\alpha+\beta$ for spot return in state 1 and 2 estimated with RSTVCJ, are respectively equal to 0.902 and 0.814 and $\alpha+\beta$ for futures return in state 1 and 2 estimated with RSTVCJ are respectively equal to 0.884 and 0.923 . Figure 1 shows the state-dependent jump volatility of jet fuel returns estimated with RSTVCJ. State 2 has higher jump volatility than state 1 . The regular correlations, namely the correlation without considering the jump component, of spot and futures returns in state 1 and 2 estimated with RSTVCJ are equal to 0.930 and 0.786 , 
respectively. Figure 2 shows the state-dependent correlation with RSTVCJ. State 1 has higher correlation than state 2 . Figure 3 is the regime probability of being in state 1 estimated with RSTVCJ.

The jump intensity is measured with the variable of $\lambda^{\prime} s$. For a given $a+b$, higher $\lambda$ implies higher expected number of jumps. The $\lambda^{\prime} s$ estimated with RSTVCJ for jet fuel spot are equal to 0.009 and 0.110 for state 1 and 2, respectively and the $\lambda$ 's estimated with RSTVCJ for crude oil futures are equal to 0.349 and 0.351 for state 1 and 2, respectively. Figure 4 shows the state-dependent jump intensity of jet fuel returns estimated with RSTVCJ. State 2 has higher mean expected jump for jet fuel returns.

Table III presents the out-of-sample hedging effectiveness of alternative models including ordinary least square $(O L S)$, constant correlation model (CC, Bollerslev, 1988), the common jump model $(C J)$ which has a jump dynamic restricted to be the same for both spot and futures returns, the state-independent correlated jump model (SICJ) with no regime switching effect in the correlated jump dynamic, the regime switching correlated jump model $(R S C J)$ without autoregressive jump intensity dynamic and the regime switching time-varying correlated jump (RSTVCJ) model. We find that RSCJ exhibits superior out-of-sample hedging performance. Figure 5 shows the estimates of hedge ratios estimated with dynamic RSTVCJ model and static OLS. method

The unhedged out-of-sample variance is 41.333. If hedger applies static OLS hedging, the variance is reduced to 6.273 or an $84.75 \%$ variance reduction. Without considering the jump dynamic, the percentage variance reductions is $84.37 \%$ for $C C$ hedging strategies. When we take account of the common jumps between spot and futures return and assume a time-invariant jump dynamic, $C J$ has a percentage variance reduction of $84.16 \% \%$. We find that $C C$ and $C J$ do not provide superior performance compared with the static OLS hedging. We consider three correlated jump models in this paper. The percentage variance reduction of SICJ is equal to $84.96 \%$. Allowing the jumps to be correlated improves the crude oil futures hedging effectiveness. When we further incorporate regime switching dynamic into the jump dynamic, the $R S C J$ hedging has an $85.67 \%$ variance reduction. Taking account of the regime switching effect improves cross hedging effectiveness. If we allow the jump dynamic to be time-varying with an autoregressive jump intensity as shown in equation (17), the RSTVCJ has a percentage variance reduction of $85.04 \%$. Taking the time-varying jump intensity dynamic into consideration does not further increase the hedging performance. $R S C J$ is the best performer and the improvements of RSCJ over CC, CJ, SICJ and RSTVCJ are $1.3 \%, 1.51 \%, 0.71 \%$ and $0.63 \%$, respectively. Overall, a hedging model taking account of both regime switching and correlated jumps improves crude oil futures hedging effectiveness.

To better understand the economic significance of the superiority of RSTVCJ over alternative models, he expected utility gains is also reported in Table III. In line with previous research (Alizadeh and Nomikos, 2004; Lee 2009, 2010; Sheu and Lee, 2014), the hedger is assumed to have an expected utility function given by equation (48) with the coefficient of absolute risk aversion $\kappa$ equal to 4 . If a hedger uses $O L S$ hedging, the average weekly utility is $U_{O L S}=0.155-4(6.273) \approx-24.938$. With $R S C J$ hedging, the average weekly utility is $U_{R S C J}=0.095-4(5.894) \approx-23.481$. The hedger's net benefit from using $R S C J$ hedging over 
OLS hedging is equal to $U_{R S C J}-U_{O L S}-C=1.457-C$, where $C$ stands for the extra transaction cost from dynamic rebalancing using $R S C J$ hedging over $O L S$ hedging. A meanvariance expected utility-maximizing hedger will adopt $R S C J$ hedging if the rebalancing cost is less than the utility gain of switching from $O L S$ hedging to RSCJ hedging. Because a typical round trip transaction cost is around $0.02 \%$ to $0.04 \%$ (Lee, 2010) and the utility gains of $R S C J$ hedging over all alternative models are all positive and much higher than the typical round trip transaction cost, hedger will adopt the $R S C J$ hedging.

\section{CONCLUSIONS}

The main contribution of this paper is to propose a bivariate regime switching timevarying correlated jump model (RSTVCJ) for cross futures hedging. Most of the existing discrete time hedging models assume a common jump for both spot and futures returns which might be too restrictive because spot and futures returns are normally not perfectly correlated. The propose RSTVCJ release this common jump assumption and allow the spot and futures returns to possess both self-owned and correlated jumps via a regime switching spillover factor. The RSTVCJ allows us to analyze the covariance structure of spot and futures returns by considering simultaneously many time-series features including regime switching variance and correlations, regime switching jump intensity and time-varying autoregressive jump intensity dynamic.

The usefulness of RSTVCJ is illustrated by performing an exercise on hedging jet fuel spot holding with crude oil futures. The hedging performance of RSTVCJ is compared with three nested models including the common jump model $(C J)$ which has a jump dynamic restricted to be the same for both spot and futures returns, the state-independent correlated jump model $(S I C J)$ with no regime switching effect in the correlated jump dynamic and the regime switching correlated jump model (RSCJ) without autoregressive jump intensity dynamic. Empirical results show that $R S C J$ has the best out-of-sample hedging performance. $R S C J$ is the best performer and the improvements of RSCJ over CJ, SICJ and RSTVCJ are $1.51 \%, 0.71 \%$ and $0.63 \%$, respectively. Generally speaking, a hedging model taking account of both regime switching and correlated jumps improves the crude oil futures hedging effectiveness. Adding time-varying autoregressive jump intensity dynamic to the jump process, however, does not further improve the futures hedging effectiveness. Results on expected utility gains of $R S C J$ over alternative models also show that a mean-variance expected utility-maximizing hedger will adopt $R S C J$ hedging even after taking account of the transaction costs. 


\section{International Conference on \\ MANAGEMENT, ECONOMICS \& FINANCE}

Table 1. Summary statistics for weekly returns of jet fuel spot and crude oil futures

\begin{tabular}{lrrrrc}
\hline & \multicolumn{2}{c}{ Jet Fuel } & & \multicolumn{2}{c}{ Crude Oil Futures } \\
\cline { 2 - 3 } \cline { 5 - 6 } Level & Log Return & & Level & Log Return \\
\hline Mean & 138.78 & 0.02 & & 48.97 & 0.02 \\
Maximum & 416.38 & 27.70 & & 144.26 & 21.94 \\
Minimum & 29.88 & -29.54 & & 9.98 & -38.45 \\
Std. Dev. & 96.14 & 5.02 & & 35.26 & 4.47 \\
Skewness & 0.75 & -0.33 & & 0.78 & -0.74 \\
Kurtosis & 2.19 & 6.74 & & 2.19 & 8.80 \\
Jarque-Bera & $156.68^{* * *}$ & $784.54^{* * *}$ & & $167.39^{* * *}$ & $1948.85^{* * * *}$ \\
\hline
\end{tabular}

Note: $* * *$ indicates significance at the $1 \%$ level and returns are calculated as the differences in the logarithm of prices multiplied by 100 . 
International Conference on

MANAGEMENT, ECONOMICS \& FINANCE

Table 2. Estimates of unknown parameters for RSTVCJ, RSCJ and SICJ models

\begin{tabular}{|c|c|c|c|c|c|c|c|}
\hline & SICJ & $R S C J$ & RSTVCJ & & $S I C J$ & $R S C J$ & RSTVCJ \\
\hline & \multicolumn{3}{|c|}{ Volatility Equation } & & \multicolumn{3}{|c|}{ Jump Intensity } \\
\hline \multirow[t]{2}{*}{$\gamma_{c 1}$} & 0.000 & 0.251 & 0.212 & $\lambda_{c 1}$ & 1.072 & 0.000 & 0.009 \\
\hline & $(0.023)^{1}$ & $(0.500)$ & $(0.156)^{*}$ & & $(0.694)^{*}$ & $(0.009)$ & $(0.020)$ \\
\hline \multirow[t]{2}{*}{$\gamma_{c 2}$} & 9.034 & 4.869 & 5.022 & $\lambda_{c 2}$ & & 0.076 & 0.110 \\
\hline & $(8.850)$ & $(1.208)^{* * *}$ & $(3.016)^{*}$ & & & $(0.050)^{*}$ & $(0.108)$ \\
\hline \multirow[t]{2}{*}{$\gamma_{f 1}$} & 0.764 & 1.258 & 0.925 & $\lambda_{f 1}$ & 1.939 & 1.697 & 0.349 \\
\hline & $(0.690)$ & $(0.462) * * *$ & $(0.718)^{*}$ & & (1.818) & $(2.292)$ & $(0.565)$ \\
\hline \multirow[t]{3}{*}{$\gamma_{f 2}$} & & & & & & & \\
\hline & 3.558 & 1.393 & 2.697 & $\lambda_{f 2}$ & & 0.641 & 0.351 \\
\hline & (3.496) & $(0.696)^{* *}$ & $(1.609)^{* *}$ & & & $(0.778)$ & $(0.364)$ \\
\hline \multirow[t]{2}{*}{$\alpha_{c 1}$} & 0.085 & 0.070 & 0.081 & & \multicolumn{3}{|c|}{ Time-Varying Jump Dynamic } \\
\hline & $(0.031)^{* * *}$ & $(0.031)^{* *}$ & $(0.060)^{*}$ & $a_{c 1}$ & & & 0.342 \\
\hline \multirow[t]{3}{*}{$\alpha_{c 2}$} & & & & & & & \\
\hline & 0.181 & 0.114 & 0.065 & & & & $(0.426)$ \\
\hline & $(0.065)^{* * *}$ & $(0.034)^{* * *}$ & $(0.069)$ & $a_{c 2}$ & & & 0.747 \\
\hline \multirow[t]{2}{*}{$\alpha_{f 1}$} & 0.202 & 0.178 & 0.197 & & & & $\begin{array}{c}(0.243)^{* *} \\
*\end{array}$ \\
\hline & $(0.061)^{* * *}$ & $(0.033)^{* * *}$ & $(0.056)^{* * * *}$ & $b_{c 1}$ & & & 0.342 \\
\hline \multirow[t]{3}{*}{$\alpha_{f 2}$} & & & & & & & \\
\hline & 0.017 & 0.021 & 0.028 & & & & $(0.603)$ \\
\hline & $(0.043)$ & $(0.033)$ & $(0.058)$ & $b_{c 2}$ & & & 0.747 \\
\hline \multirow[t]{2}{*}{$\beta_{c 1}$} & 0.803 & 0.860 & 0.821 & & & & $(0.413) * *$ \\
\hline & $(0.048)^{* * *}$ & $(0.065)^{* * *}$ & $(0.111)^{* * * *}$ & $a_{f 1}$ & & & 0.906 \\
\hline \multirow[t]{3}{*}{$\beta_{c 2}$} & & & & & & & $(0.324)$ \\
\hline & 0.589 & 0.707 & 0.749 & & & & $* * *$ \\
\hline & $(0.203)^{* * *}$ & $(0.065) * * *$ & $(0.215)^{* * * *}$ & $a_{f 2}$ & & & 0.416 \\
\hline \multirow[t]{2}{*}{$\beta_{f 1}$} & 0.692 & 0.715 & 0.687 & & & & $(0.432)$ \\
\hline & $(0.048)^{* * *}$ & $(0.054)^{* * *}$ & $(0.061)^{* * *}$ & $b_{f 1}$ & & & 0.906 \\
\hline \multirow[t]{4}{*}{$\beta_{f 2}$} & & & & & & & \\
\hline & 0.907 & 0.951 & 0.895 & & & & $(0.993)$ \\
\hline & $(0.077)^{* * *}$ & $(0.048)^{* * * *}$ & $(0.120)^{* * *}$ & $b_{f 2}$ & & & 0.136 \\
\hline & & elation Equa & & & & & $(0.079)^{* *}$ \\
\hline \multirow[t]{2}{*}{$\rho_{1}$} & 0.944 & 0.914 & 0.930 & & & over facto & \\
\hline & $(0.054)^{* * *}$ & $(0.015)^{* * *}$ & $(0.019)^{* * *}$ & $\eta_{1}$ & 0.719 & -1.182 & -0.686 \\
\hline \multirow[t]{3}{*}{$\rho_{2}$} & 0.634 & 0.712 & 0.786 & & $(0.273)^{* * * *}$ & $(1.323)$ & (1.335) \\
\hline & $(0.082)^{* * * *}$ & $(0.030)^{* * *}$ & $(0.037)^{* * *}$ & $\eta_{2}$ & & 0.384 & 0.053 \\
\hline & \multicolumn{3}{|c|}{ Jump Distribution } & & & $(0.378)$ & $(0.047)$ \\
\hline
\end{tabular}




\section{International Conference on}

\section{MANAGEMENT, ECONOMICS \& FINANCE}

\begin{tabular}{cccc}
\cline { 3 - 4 }$\tau_{c}$ & 0.229 & 0.226 & 0.373 \\
& $(0.240)$ & $(0.806)$ & $(0.535)$ \\
$\delta_{c}$ & 1.206 & 9.013 & 4.601 \\
& $(0.312)^{* * *}$ & $(2.839)^{* * *}$ & $(0.752)^{* * *}$ \\
$\tau_{f}$ & 0.720 & 0.190 & 0.381 \\
& $(0.444)^{*}$ & $(0.170)$ & $(0.241)^{*}$ \\
$\delta_{f}$ & 0.010 & 0.000 & 0.003 \\
& $(0.018)$ & $(0.028)$ & $(0.012)$ \\
\hline \multirow{2}{*}{$\mathrm{LL}^{2}$} & -6827.1405 & -6807.122 & -6803.56 \\
\hline
\end{tabular}

1. Figures in parentheses are standard errors and $*, * *$ and $* * *$ indicate significance at the $10 \%, 5 \%$ and $1 \%$ levels, respectively.

2. $L L$ stands for the log likelihood value. 
Table3. Out-of-sample hedging effectiveness

\begin{tabular}{|c|c|c|c|c|c|c|}
\hline & $\begin{array}{c}\text { Variance } \\
\text { of Hedged } \\
\text { Portfolio } \\
\text { Return }\end{array}$ & $\begin{array}{c}\text { Percent } \\
\text { age } \\
\text { Variance } \\
\text { Reduction }^{1}\end{array}$ & $\begin{array}{c}\text { Improv } \\
\text { ement of } \\
R S C J \text { over } \\
\text { Other } \\
\text { Models }^{2} \\
\end{array}$ & $\begin{array}{l}\text { Hedg } \\
\text { ed } \\
\text { Portfolio } \\
\text { Return } \\
\end{array}$ & $\begin{array}{r}\text { Expe } \\
\text { cted } \\
\text { Weekly } \\
\text { Utility }^{3} \\
\end{array}$ & $\begin{array}{c}\text { Utility Gain } \\
\text { of } R S C J \text { over } \\
\text { Other Models } \\
\end{array}$ \\
\hline Unhedged & 41.133 & & & & & \\
\hline$O L S$ & 6.273 & $84.75 \%$ & $0.92 \%$ & 0.155 & $\begin{array}{c}- \\
24.938\end{array}$ & 1.457 \\
\hline$C C$ & 6.427 & $84.37 \%$ & $1.30 \%$ & 0.165 & 25.543 & 2.063 \\
\hline$C J$ & 6.516 & $84.16 \%$ & $1.51 \%$ & 0.146 & 25.918 & 2.437 \\
\hline SICJ & 6.186 & $84.96 \%$ & $0.71 \%$ & 0.106 & $\begin{array}{c}- \\
24.637\end{array}$ & 1.156 \\
\hline$R S C J$ & 5.894 & $85.67 \%$ & & 0.095 & 23.481 & \\
\hline RSTVCJ & 6.154 & $85.04 \%$ & $0.63 \%$ & 0.148 & $\begin{array}{c}- \\
24.468 \\
\end{array}$ & 0.987 \\
\hline
\end{tabular}

1. Percentage variance reductions are calculated as the differences of the variance of unhedged position and the estimated variances of alterative models over the variance of unhedged position multiplied by 100.

2. Improvement of RSCJ over other hedging strategies is defined as the differences of the percentage variance reduction of $R S C J$ and the percentage variance reduction of alternative models.

3. Expected weekly utility is calculated based on equation (48)

Utility gains of RSCJ over other hedging strategies are defined as the differences of the expected utility of $R S C J$ and the expected utilities of alternative models. 


\section{International Conference on MANAGEMENT, ECONOMICS \& FINANCE}

Figure1. State dependent jump volatility of jet uel returns estimated with RSTVCJ

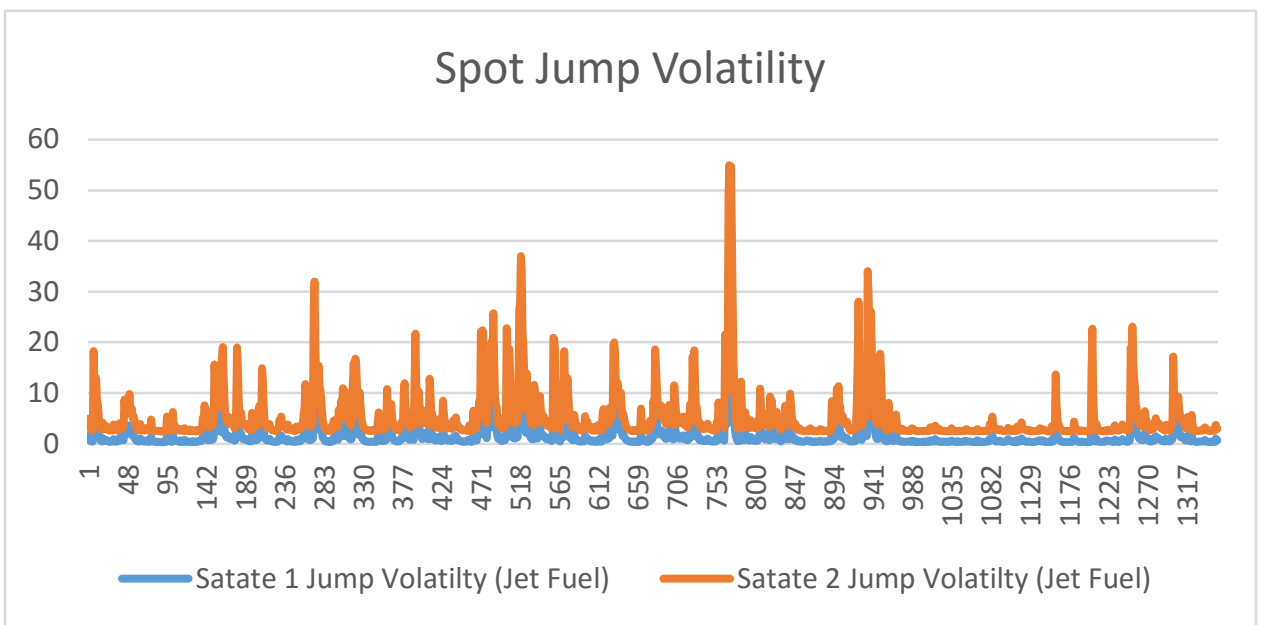

Figure 2. State-dependent correlation estimated with RSTVCJ

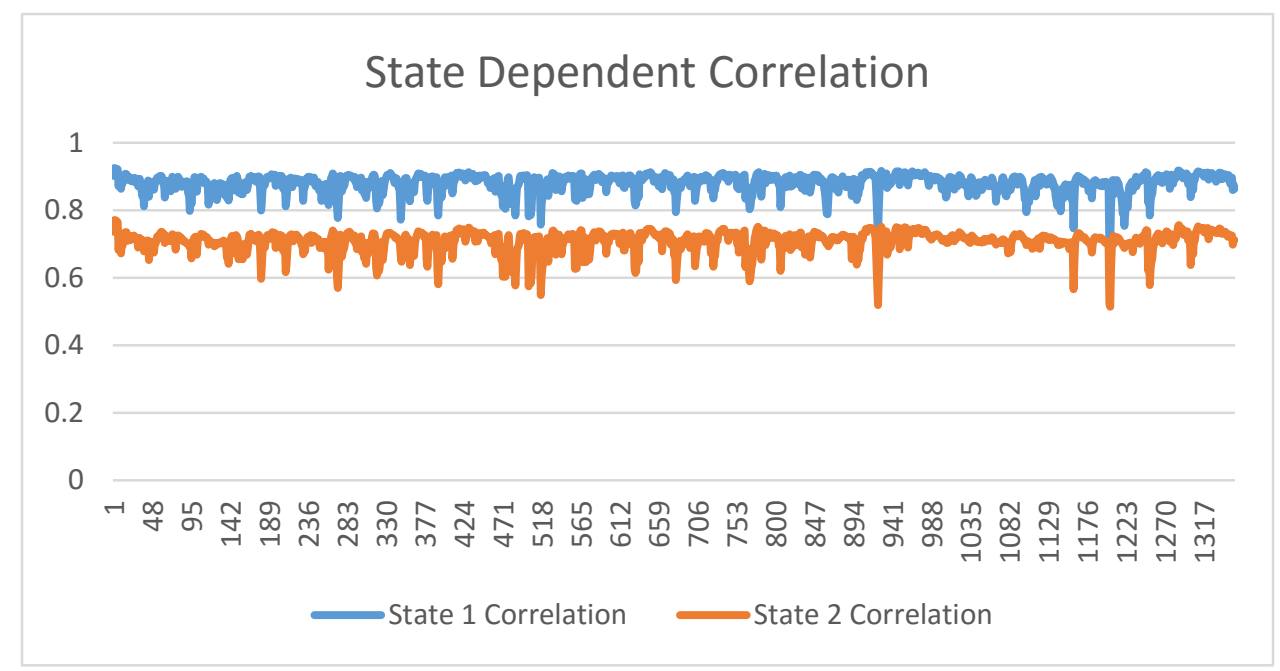

Figure 3. Regime probability of being in state 1 estimated with RSTVCJ 


\section{International Conference on MANAGEMENT, ECONOMICS \& FINANCE}

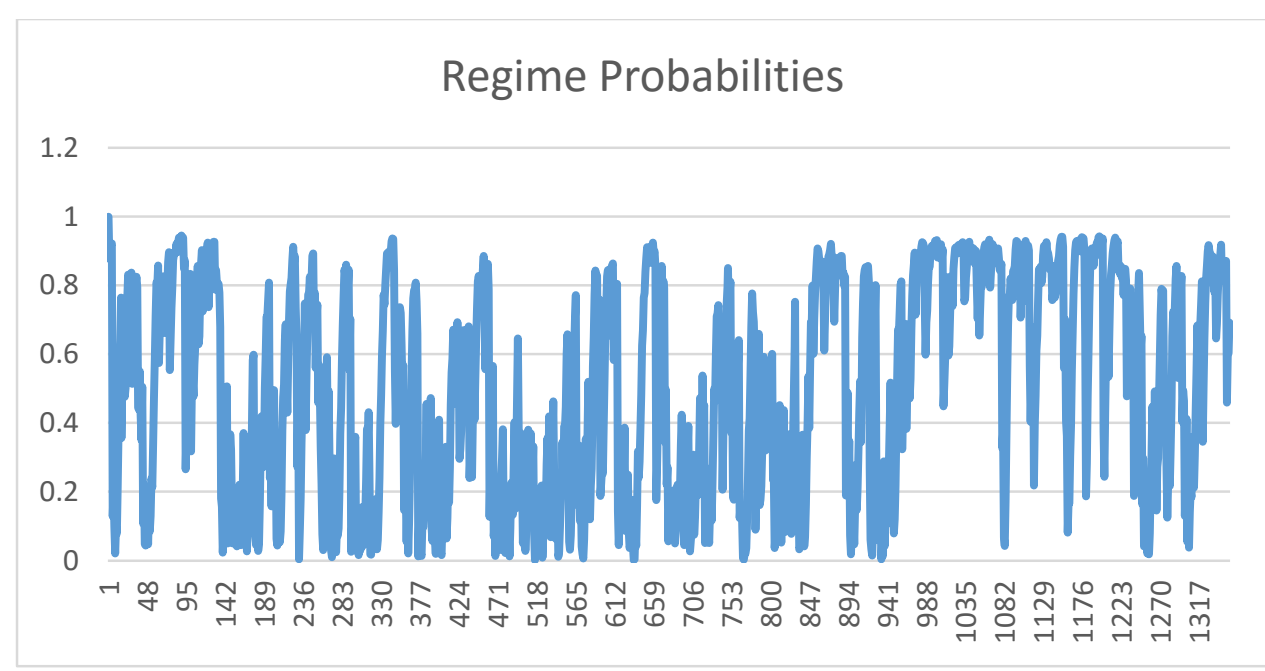

Figure 4. State dependent jump intensity of jet fuel returns estimated with RSTVCJ

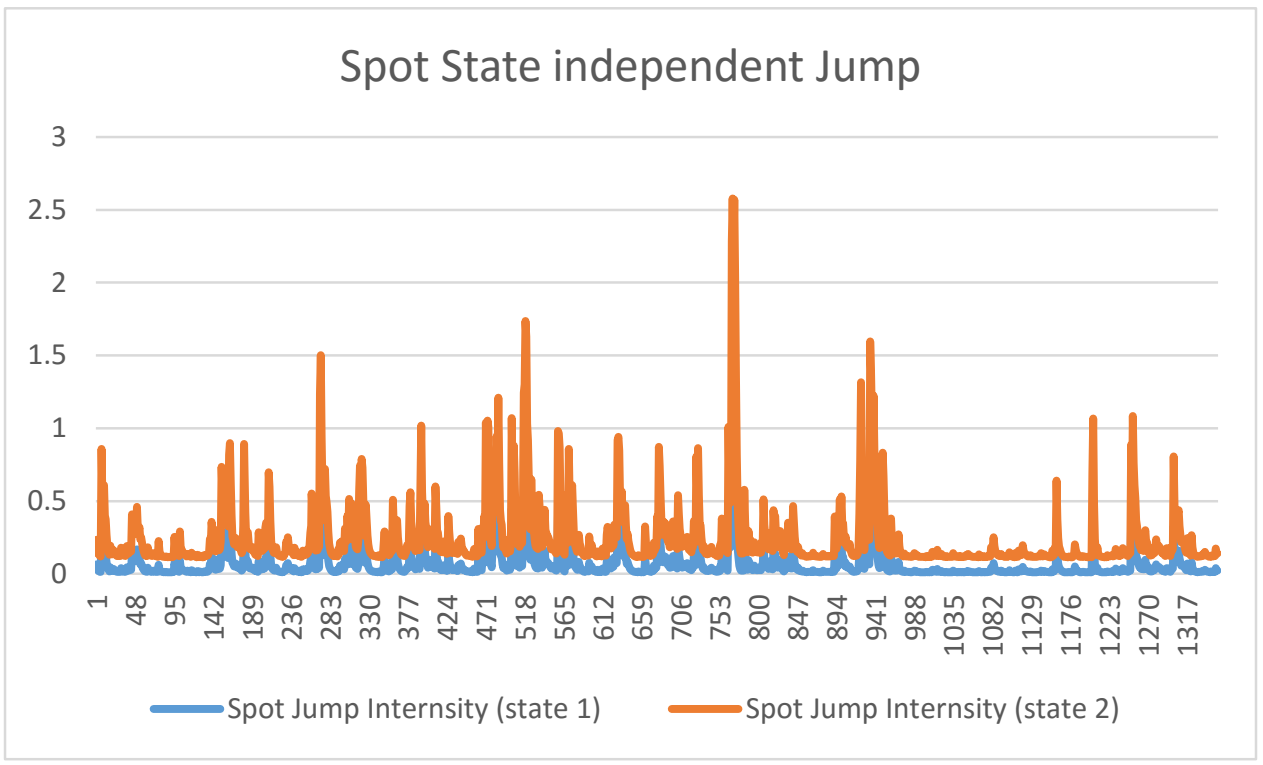

Figure 5. Hedge ratio estimated with OLS and RSTVCJ 


\section{International Conference on MANAGEMENT, ECONOMICS \& FINANCE}

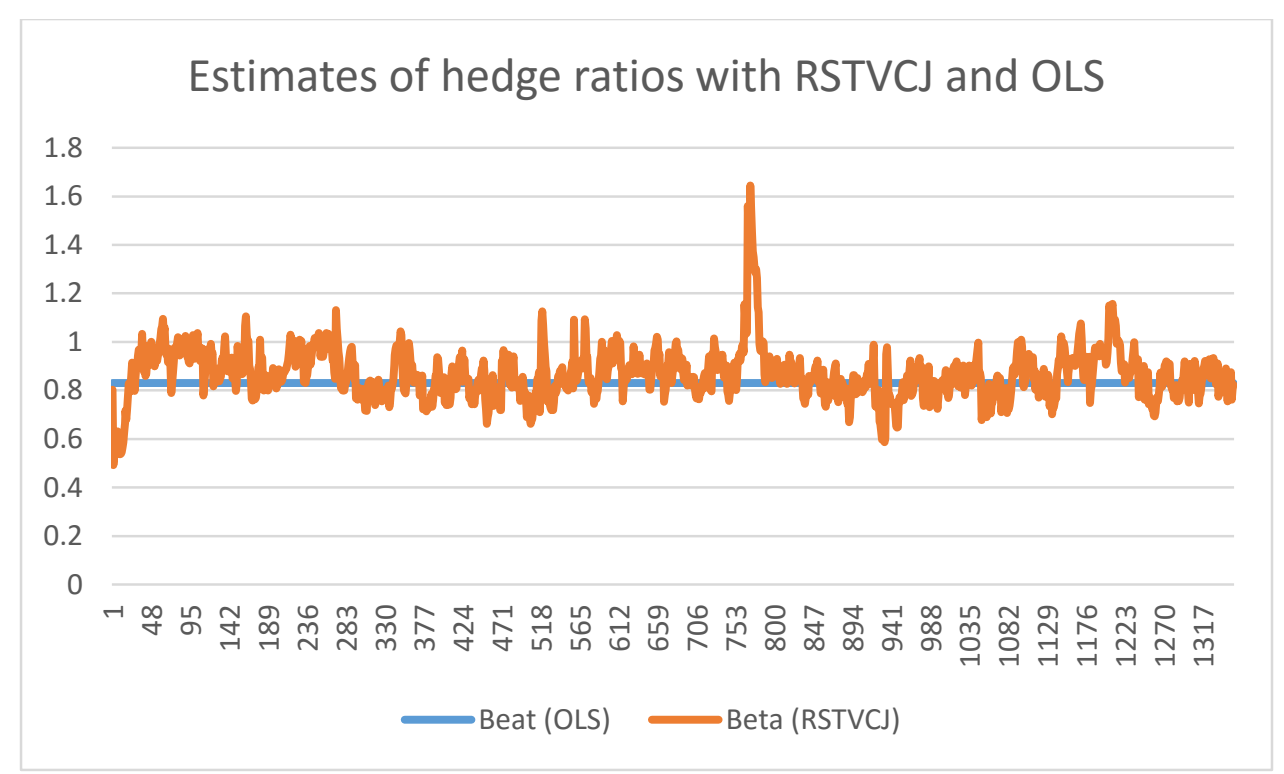

\section{Acknowledgment}

The author would like to thank the National Science Council, Taiwan, ROC, for supporting this research under Grant No. NSC- 105-2410-H-260 -013 


\section{References}

[1] Brooks, C., Henry, O. T., Persand, G., 2002. The effect of asymmetries on optimal hedge ratios. Journal of Business 75, 333-352.

[2] Cai, J., 1994. A Markov model of switching-regime ARCH. Journal of Business and Economic Statistics 12, 309-316.

[3] Chan, W. H., 2003. A correlated bivariate poisson jump model for foreign exchange. empirical economics 28, 669-689.

[4] Chan, W. H., 2008. Dynamic hedging with foreign currency futures in the presence of jumps. Studies in Nonlinear Dynamics and Econometrics 12, art4.

[5] Chan, W. H., 2010. Optimal hedge ratios in the presence of common jumps. Journal of Futures Markets 30, 801-807.

[6] Chan, W. H., and Maheu, J. M., 2002. Conditional jump dynamics in stock market returns. Journal of Business \& Economic Statistics 20, 377-389.

[7] Chan, W. H., and Young, D., 2006. Jumping hedges: an examination of movements in copper spot and futures markets. The Journal of Futures Markets 26, 169-188.

[8] Chan, W. H. and Young, D., 2009. Conditional jump dynamics for copper prices”, Review of Futures Markets 18, 75-85.

[9] Diebold, F.X., Mariano, R.S., 1995. Comparing predictive accuracy. Journal of Business and Economic Statistics 13, 253-263.

[10] Ederington, L. H., Salas, J. M., 2008. Minimum variance hedging when spot price changes are partially predictable $32,654-663$.

[11] Gagnon, L., Lypny, G., McCurdy, T., 1998. Hedging foreign currency portfolios. Journal of Empirical Finance 5, 197-220.

[12] Gray S. F., 1996. Modeling theconditional distribution of interest rates as a regimeswitching process. Journal of Financial Economics 42, 27-62.

[13] Hamilton, J. D., 1989. A new approach to the economic analysis of nonstationary time series and the business cycle. Econometrica 57, 357-384.

[14] Hamilton, J. D., Susmel, R., 1994. Autoregressive conditional heteroscedasticity and changes in regime. Journal of Econometrics 64, 307-333.

[15] Kroner, K. F., Sultan J., 1993. Time-varying distribution and dynamic hedging with foreign currency futures. Journal of Financial and Quantitative Analysis 28, 535-551.

[16] Lee, H. T., 2009a. Optimal futures hedging under jump switching dynamics. Journal of Empirical Finance16, 446-456.

[17] Lee, H. T., 2009b. A copula-based regime-switching GARCH model for optimal futures hedging. Journal of Futures Markets 29, 946-972.

[18] Lee, H. T., 2010. Regime switching correlation hedging. Journal of Banking \& Finance 34, 2728-2741. 
[19] Lee, H. T., 2015. A Volatility spillover hedging model with multi-chain regime switching jump dynamic. Working paper.

[20] Lee, H. T., Ko, K. C., 2010. Cross hedging effectiveness of Taiwan stock index futures. Journal of Futures and Options 3, 33-55.

[21] Lee, H. T., Tsang, W. L., 2011. Cross hedging single stock with American Depositary Receipt and stock index futures. Finance Research Letters 8, 146-157.

[22] Lee, H. T., Yoder, J. K., 2007a. A Bivariate Markov Regime Switching GARCH Approach to Estimate the Time Varying Minimum Variance Hedge Ratio. Applied Economics 39, 1253-1265.

[23] Lee, H. T., Yoder, J. K., 2007b. Optimal hedging with a regime-switching time-varying correlation GARCH Model. The Journal of Futures Markets 27, 495-516.

[24] Lee, H. T., Yoder, J. K., Mittelhammer, R. C., McCluskey, J. J., 2006. A Random Coefficient Autoregressive Markov Regime Switching Model for Dynamic Futures Hedging. The Journal of Futures Markets 26, 103-129.

[25] McCracken, M., 2007. Asymptotics for out-of-sample tests of Granger causality. Journal of Econometrics 140, 719-752.

[26] Park, T. H., Switzer, L. N., 1995. Bivariate GARCH estimation of the optimal hedge ratios for stock index futures: A Note. The Journal of Futures Markets 15, 61-67.

[27] Ratner, M., Chiu, C. C., 2013. Hedging stock sector risk with credit default swaps. International Review of Financial Analysis 30, 18-25.

[28] Sarno, L., and Valente, G., 2000. The Cost of Carry Model and Regime Shifts in Stock Index Futures Markets: An Empirical Investigation. The Journal of Futures Markets 20, 603-624.

[29] Sarno, L., and Valente, G., 2005a. Empirical Exchange Rate Models and Currency Risk: Some Evidence from Density Forecasts. Journal of International Money and Finance 24, 363-385.

[30] Sarno, L., and Valente, G., 2005b. Modelling and Forecasting Stock Returns: Exploiting the Futures Market, Regime Shifts, and International Spillovers. Journal of Applied Econometrics 20, 345-376.

[31] Sheu, H. J., Lee, H. T., 2012. A full jump switching level GARCH model for short-term interest rate. Applied Financial Economics 22, 479-489.

[32] Sheu, H. J., Lee, H. T., 2014. Optimal futures hedging under multi-chain Markov regime switching, Journal of Futures Markets 34, 173-202.

[33] Sheu, H. J. Lee, W. C., Lee, H. T., 2015. The cross hedging effectiveness of oil futures for non-energy commodities under regime switching, Journal of Futures and Options 8, 4184.

West, Kenneth D., 1996. Asymptotic inference about predictive ability. Econometrica 64, 1067-1084. 